\title{
Detecting common longevity trends by a multiple population approach
}

\author{
Valeria D’Amato $^{1}$, Steven Haberman ${ }^{2}$, Gabriella Piscopo $^{3}$, Maria Russolillo ${ }^{1}$, Lorenzo Trapani ${ }^{4}$ \\ 1 Department of Economics and Statistics, University of Salerno, via Ponte Don Melillo, Campus \\ Universitario, 84084 Fisciano (Salerno), Italy \\ e-mail:vdamato@unisa.it,mrussolillo@unisa.it
}

2 Faculty of Actuarial Science and Insurance, Cass Business School, City University London,

Bunhill RowLondon, UK, e-mail: s.haberman@city.ac.uk,

${ }^{3}$ Department of Economics, University of Genoa, Via Vivaldi, 16126 Genoa, Italy - e-mail:

piscopo@economia.unige.it

4 Faculty of Finance, Cass Business School, City University London, Bunhill Row London, UK, e-

mail:L.Trapani@city.ac.uk

\begin{abstract}
Recently the interest in the development of country and longevity risk models (Njienga and Sherris. 2011) has been growing. The investigation of long-run equilibrium relationships could provide valuable information about the factors driving changes in mortality, in particular across ages and across countries. In order to investigate cross-country common longevity trends, tools to quantify, compare and model the strength of dependence become essential. On one hand, it is necessary to take into account either the dependence for adjacent age groups, or the dependence structure across time in a single population setting: a sort of intradependence structure (D'Amato et al. 2012b). On the other hand, the dependence across multiple populations, which we describe as inter-dependence, can be explored for capturing common long run relationships between countries. The objective of our work is to produce longevity projections by taking into account the presence of various forms of cross-sectional and temporal dependencies in the error processes of multiple populations, considering mortality data from different countries. The algorithm that we propose combines model-based predictions in the Lee Carter (LC) framework (1992) with a bootstrap procedure for dependent data, and so both the historical parametric structure and the intra-group error correlation structure are preserved. We modify the model presented by D'Amato et al. (2012b), which applies a sieve bootstrap to the residuals of the LC model and is able to reproduce, in the sampling, the dependence structure of the data under consideration. In the current paper, the algorithm that we build is applied to a pool of populations by using ideas from panel data; we refer to this new algorithm as the Multiple Lee Carter Panel Sieve (MLCPS). We are interested in estimating the relationship between populations of similar socioeconomic conditions. The empirical results show that the MLCPS approach works well in the presence of dependence.
\end{abstract}

Keywords: Serial and Cross-sectional Correlation, Factor Models, Vector Autoregression, Sieve Bootstrap, Lee Carter model

\section{Introduction}


In the actuarial literature and practice, the interest in the development of country longevity risk models is increasing (Njenga and Sherris, 2011). Attention is focused on investigating long-run equilibrium relationships and collecting valuable information about the factors driving the changes in mortality, in particular across ages and countries. The importance of considering a synoptic approach has been highlighted by Li and Lee (2005). They show an improvement in the mortality projections for individual countries through taking into account the patterns in a larger group. Thus, Tuljapurkar et al. (2000) identify a 'universal pattern' of mortality decline by analysing together the seven richest countries. Similarly, although using a different model, Russolillo et al. (2011) provide aggregate estimates for a set of countries. The underlying idea is to produce projected life tables for the set of countries under consideration.

Lazar and Denuit (2009) extract and forecast the common stochastic trends shared by the time series of log-death rates, where the common factors can be modelled as a multivariate random walk with drift. To hedge against the basis risk, Li and Hardy (2011) connect the longevity improvements of two different populations by the Augmented Common Factor Model in the Lee Carter (LC) setting. They show that using two independent LC models is likely to result in an increasing divergence in life expectancy in the long run. This is counter to a global convergence in mortality levels, as documented in White (2002), Wilson (2001), and United Nations (1998).

In order to investigate cross-country common longevity trends, we adopt a multiple population approach. For the purposes under consideration, the dependence structure has to be taken into account, in order to avoid an underestimation of the actual mortality risk. Indeed, the pressing need for the correct representation of the longevity phenomenon is also more relevant in light of the new insurance regulatory guidelines prescribed by Solvency II. In order to produce accurate longevity projections, it is essential to include the so-called dependency risk, which is a significant source of risk as explained in D'Amato et al. (2011). On one hand, it is necessary to take into account either the dependence for adjacent age groups or the dependence structure across time for a single population: which is a type of intra-dependence. On the other hand, the dependence across the multiple populations under consideration has to be explored: here we describe this as interdependence.

The existence of dependence in mortality data involves the interactions between age and time. In particular, the mortality experience of countries in the industrialized world over the course of the twentieth century would suggest a substantial age-time interaction: two dominant trends have affected different age groups at different times. Booth et al. (2002) show that the interaction exists, in the application of the LC model to Australian data. Furthermore, they acknowledge that the main methodological problem in the LC model is the assumption of invariance in the age component. To overcome the problem they propose an extension of the original model. According to other authors, like Stevens (2011), the assumption that the bx component in LC is time-independent is violated. In fact, empirical evidence shows that this assumption is indeed violated. In particular, Stevens proposes a different variant to the traditional LC model, involving a time-dependent-age factor. Others have introduced switching regimes (Hainut, 2012) or multiple factor models for mortality using an affine model for all ages simultaneously (Gaille and Sherris, 2011). In particular, a multiple LC model is developed to capture the stochastic trends in mortality improvements at different ages and across time as well as a multivariate dependence structure across ages.

In this paper, we consider a different approach to the issue of cross-sectional and time dependence. We retain the parametric structure of the LC model, but we extend the basic framework to include some cross dependence in the error term. As far as time dependence is concerned, we allow for all of the idiosyncratic components (both in the common stochastic trend and in the error term) to follow a linear process, and we are thus considering a highly flexible specification for the serial 
dependence structure of our data. We also relax the assumption of normality, which is typical of early studies on mortality (Lee and Carter, 1992) and on factor models (Anderson, 1984). Whilst the technical details are in Section 4, we point out that our model nests the standard LC framework, thereby having the same properties and also being more general.

From a methodological point of view, our paper is the first one to apply bootstrap to such a general context. Although the asymptotic properties for panel factor models are well-developed (Bai, 2004), it is well known that the estimation techniques (essentially based on applying the Principal Components method) may be flawed in small samples; moreover, limiting distributions of estimated parameters depend upon several nuisance parameters whose estimation can be fraught with difficulties. Thus, the bootstrap can be, in this context, a valid alternative. By virtue of our assumption on the time dependence structure, we apply a sieve bootstrap algorithm (Bulhmann, 1997) to the Vector Auto-Regression (VAR henceforth) model containing the estimated common factors (both stationary and non-stationary). However, in our context, we cannot apply a standard sieve bootstrap algorithm, since, when resampling the estimated common factors, a generated regressors problem arises. Trapani (2012) develops an algorithm to apply sieve bootstrap to the context of non-stationary panel factor series, developing selection rules for the order of the VAR and showing the superior performance of sieve bootstrap compared to first-order asymptotics. Our paper is, therefore, the first application of the bootstrap theory for non-stationary panel factor series.

Based on this methodology, we produce longevity projections by taking into account the presence of various forms of cross-sectional and temporal dependencies in the error processes in relation to a multiple population dataset, which is composed of mortality data from different countries. (The following phrase has been cut)

The remainder of the paper is organised as follows. Section 2 introduces the LC model. In Section 3, we present the setting of multiple population on which we develop the algorithm. Section 4 is devoted to the proposed MLCPS. Section 5 shows the numerical applications. Finally, Section 6 concludes.

\section{Demographic scenario: the Lee Carter model}

For each population i, the LC (1992) model suggested a log-bilinear form for the force of mortality:

$$
\begin{aligned}
& m_{x t, i}=\exp \left(\alpha_{x, i}+\beta_{x, i} k_{t, i}+u_{x t, i}\right) \\
& y_{x t, i} \equiv \ln \left(m_{x t, i}\right)=\alpha_{x, i}+\beta_{x, i} k_{t, i}+u_{x t, i}
\end{aligned}
$$

describing the $\log$ of a time series of age-specific death rates $m_{x t, i}$ as the sum of an age-specific parameter independent of time $\alpha_{x, i}$ and a component given by the product of a time-varying parameter $k_{t, i}$, reflecting the general level of mortality and the parameter $\beta_{x, i}$, representing how rapidly or slowly mortality at each age varies when the general level of mortality changes.

We refer to Lee and Carter (1992) for a fuller discussion of the model. 


\section{Multiple Mortality Panel}

In this research, we analyse mortality datasets related to five populations experiencing common longevity improvements, in order to compare the evolution of mortality in different countries in the light of cross-country common trends. Working with different populations, the dependence structure that has been analyzed in previous works for a single dataset (D'Amato et al., 2012a) becomes very complex and has to be taken into account under a multidimensional approach. In fact, the cross sectional dependence for adjacent age groups, across countries and serial/time dependence needshave to be considered. In this case, the classical VAR sieve bootstrap framework appears infeasible and cannot be applied to our three-dimensional dataset, because the number of crosssectional units is too large. Thus, we present an original contribution to overcome the problem of analyzing dependence in the case of multiple populations.

Our research starts from the idea that the common trends between countries are captured by the parameters $k_{t}$ of the LC model. For this reason, we fit separately the LC to some mortality data for $M$ different populations, composed by the same ages $x=a, a+1, \mathrm{~K}, a+N$ and years $t=b, b+1, \mathrm{~K}, b+T$, where $a$ represents the first age and $b$ the first time, respectively. Once we have obtained the $k_{t}$ 's for each country, we arrange the $M$ time series of $k_{t}$ in a matrix, generating a panel dataset in which the single units are represented by the different populations and are collected in rows. It is clear that the approach is completely different from the previous one: in the case of one population, each single unit is represented by a different age; the variable observed is the central mortality death rate and the observations are $N T$, consisting of time series of length $T$, on $N$ parallel age-units. Instead, in the case of multiple populations, each single unit is represented by a different population; the variable observed is the parameter $k_{t}$ of that particular population, which is able to explain the mortality trend; thus the observation are $M T$, consisting of $M$ time series of length $T$, one for each population. On this reduced dataset, it is possible to implement the $V A R$ sieve scheme.

\section{Algorithm: Multiple Lee Carter Panel Sieve}

This section discusses the methodology to generate the bootstrap sample.

We consider the problem of making reliable confidence intervals for projections obtained in the context of dependent data. The asymptotic analysis on the consistency and good convergence properties of the sieve bootstrap is summarized by Hardle et al. (2003) and Horowitz (2001). When it is possible to approximate the error process by an autoregression, the sieve bootstrap has properties that are superior to other simulation approach for dependent data, as the block bootstrap (Hardle et al. 2003).

For nonstationary panel factor series as a multiple population panel, we implement the sieve bootstrap, since these confidence intervals are more accurate than the asymptotic ones (Trapani 2013).

In the following we describe the algorithm to implement the sieve bootstrap in the Lee Carter framework.

The preliminary step is the construction of a matrix $\mathrm{K}$ through the fitting of the LC model to the different populations separately.

For each population i, we fit the LC model: 
$y_{x t, i}=\alpha_{x, i}+\beta_{x, i} k_{t, i}+u_{x t, i} \quad i=1, \ldots, M$

The parameters $k_{t, i}$ are extracted from the mortality rates of the i-th population by applying the Principal Components estimator to the matrix $Y_{i} Y_{i}{ }^{\prime}$, where the $Y_{i}$ is the log death rate matrix for the i-th population. In particular, after extracting the eigenvalues and eigenvectors of $Y_{i} Y_{i}^{\prime}$ and sorting them based on the magnitude of the eigenvalue in descending order, then $k_{t i}$ is estimated by the first eigenvector of $Y_{i} Y_{i}^{\prime}$ multiplied by $T$.

Having determined the estimated $k_{t i}$, say $\hat{k}_{t i}$, the $\beta_{x i} \mathrm{~s}$ are estimated applying Ordinary Least Squares (OLS) Estimation:

$\hat{\beta}_{x, i}=\left[\sum_{t=1}^{T} \hat{k}_{t, i} \hat{k}_{t, i}^{\prime}\right]^{-1}\left[\sum_{t=1}^{T} \hat{k}_{t, i} y_{t, i}\right]=\frac{1}{T} \sum_{t=1}^{T} \hat{k}_{t, i} y_{t, i}$

The parameters $k_{t, i}$ are then arranged in a matrix $\mathrm{K}_{\mathrm{MXT}}$. A VAR is fitted to this matrix. The VAR is the statistical tool employed to represent how the different populations are related each other.

Let $K_{t}=\left[k_{1} t^{\prime}, \ldots, k_{M t^{\prime}}\right]^{\prime}$ denote an $(\mathrm{Mx} 1)$ vector of time series variables. The basic q-lag VAR(q) has the form:

$K_{t}=\sum_{j=1}^{q} A_{j} K_{t-j}+\varepsilon_{t}, \quad t=1, \ldots, T$

where $A$ is the matrix of the coefficients of the selected VAR(q) model

Hence, the bootstrapping algorithm is as follows:

Step 1. (PC estimation)

(1.1)For each $i$, estimate the $k_{t, i}$ in (3) using PC.

(1.2)Arrange the fitted $k_{t, i}$ in the matrix $\mathrm{K}$

Step 2. (VAR model estimation)

(2.1) Estimate the matrix of coefficient $A$ of the VAR(q) model by applying OLS to (4). The $\mathrm{q}$ lag selection criterion is based on Akaike's information criterion (AIC)

(2.2) Compute the residuals $\hat{e}_{t, q}=\Delta \hat{k}_{t}-\sum_{j=1}^{q K} \hat{A}_{q, j} \Delta \hat{k}_{t-j}$ and centre them around their mean, defining them $\bar{e}_{t, q}$.

Step 3. (bootstrap) for $b=1, \ldots, \beth$ iterations

(3.1) (resampling) 
(3.1.a) Draw (with replacement) $T$ values from $\left\{\bar{e}_{t, q}\right\}_{t=1}^{T}$ to obtain the bootstrap sample $\left\{e_{t, b}\right\}_{t=1}^{T}$

(3.2) (generation of the bootstrap sample)

$$
\begin{aligned}
& \text { (3.2.a) } k_{t, b}=\sum_{j=1}^{q K} \hat{A}_{q, j} \Delta k_{t-j, b}+e_{t, b} \\
& \Delta \text { recursively the pseudo sample }
\end{aligned}
$$$$
\text { (3.2.b) Generate } k_{t, b} \text { as } k_{t, b}=k_{0, b}+\sum_{j=1}^{t} \Delta k_{j, b} \text {, with initialisation } k_{0, b}=k_{0} \text {. }
$$

In this way we obtain the matrix $K_{M X T}^{b}$ for each b sample from which we can generate the $\left\{y_{x t, i}^{b}\right\}_{t=1}^{T}$ as $y_{x t, i}^{b}=\hat{\alpha}_{x, i}+\hat{\beta}_{x, i} k_{t, i}^{b}$.

\section{Numerical Application}

In the present section, we provide an assessment of longevity risk by analysing the trends in the historical longevity data across ages for several countries which are expected to have experienced common longevity improvements, on the basis of similar socio-economic features and experience. In particular, the analysis considers the following countries: United Kingdom (henceforth UK), France, Italy, Spain, Belgium. The study is performed for each country on the total population (composed by male and female) ranging from 1950 to 2006, for ages from 0 up to 110 years, considered by single calendar year and by single year of age, where the class of age above 100 years is collected in an open age group, 100+. The numerical application is performed according to three phases:

1) Fitting the LC model,

2) Measuring Dependence Structure,

3) Projecting mortality.

\subsection{Fitting the LC model}

In the first phase of the numerical applications, we fit the LC model on the datasets of the five selected countries and then we identify the structure of the residuals through the traditional measures. In Figure 1, we show the estimates of the model parameters obtained by the fitting of the LC model for the five considered countries: 
Observing Figure 1, we note that the estimated values of the parameter $\alpha_{x}$ are quite similar for each country: the average of the mortality rate calculated during the years for a given age does not show significant differences across countries. Moreover, we can deduce from the fitting of the parameter $k_{t}$ that all countries have experienced a decreasing mortality trend during the years, with a small difference in the rate of reduction. For example, Italy shows a lower rate of mortality reduction until 1980 and afterwards it experiences an acceleration. In contrast, greater differences are recognized in the fitting of the parameter $\beta_{x}$, which represents how different ages react to the reduction in mortality. There is a greater variability between age 30 to 100 in the Spanish dataset and between age 0 to 20 in the Belgian dataset. This feature has an impact on the percentage of the variation explained in fitting the model, that is respectively: $93.3 \%$ for UK, $93.7 \%$ for France, $94.7 \%$ for Italy, $92 \%$ for Spain and $88.6 \%$ for Belgium. Furthermore, for each country, we compute the error measures for the fitted mortality rates. In Table 1, we show the error measure findings with various indexes of the fitting accuracy averaged across years (ME=mean error, MSE=mean squared error, $\mathrm{MPE}=$ mean percentage error and $\mathrm{MAPE}=$ mean absolute percentage error), and also whose integrated across ages (IE=integrated error, ISE=integrated squared error, IPE=integrated percentage error and IAPE=integrated absolute percentage error):

Table 1 - Error measures based on mortality rates

\subsection{Measuring Dependence Structures}

In the second stage of the numerical application, we measure either the dependence within each single population, or the dependence between different populations: respectively the intradependence and the inter-dependence. In this respect, by focusing on the intra-dependence, we include graphical analysis on autocorrelation functions by age and time, formal statistical tests as the Ljung-Box test based on the autocorrelation plot and Pearson test of independence. Regarding the inter-dependence, we investigate the long-run relationships between countries by using a VAR scheme. Regarding the intra-dependence, broadly speaking the empirical evidence confirms a dependence structure for each country. The graphical analysis is supported also by the results of Ljung-Box test, implemented for each age and for each country separatelyOn the basis of the calculations performed for each country, we conclude that, for most ages and for each country, the null hypothesis of independence is rejected. From the p-values, i.e. the probability of making an error if we reject the null hypothesis when this is true, we note that the value is very small for each age. In other words, we can confirm the randomness of the residuals, which has already been observed in the graphical analysis.

Finally, we compute the Pearson's correlation coefficient, which assumes normality in the distribution of the residuals, and it confirms a strong positive dependence for almost all countries, except Spain and Belgium which present few negative values for some ages. For instance, figure 2 illustrates the case of Italy.

Figure 2- Pearson's correlation coefficient versus age, Italy

\subsection{Projecting mortality}

At this final stage, after having assessed the dependence structures, we apply the MLPS algorithm, described in Section 4, for the datasets of UK, France, Italy, Spain and Belgium. Figure 3 plots 
simultaneously $k_{t}$ estimates, for the total population of the five countries considered. As shown, $k_{t}$ declines roughly linearly from 1950 to 2006, especially for France and Italy.

Figure 3-kt trends- UK, France, Italy, Spain, Belgium, Total population, years: from 1950 to 2006

We fit the $V A R$ model to the $k_{t}$ of each country and calculate the residuals.

Finally, on the basis of the simulations according to the algorithm proposed in Section 4 we obtain for each period the sample average $k_{t}$. Then, we project these quantities by using ARIMA models and calculate the confidence intervals. Figures 4 displays the mean of the simulated $k_{t}$ and the projections with confidence intervals for UK, Belgium, Spain, France and Italy. It is possible to note wider confidence intervals for UK and Belgium, compared to the ones for the other three countries.

Figure 4- mean kt for UK, Belgium, Spain, France and Italy

Table 2-6 illustrate the means of the projection of $k_{t}$ for $h=1, \mathrm{~K}, 15$ periods ahead, calculated using 1000 simulations. Moreover, the $80 \%$ and $95 \%$ confidence intervals values are shown in the same tables for each country and are compared with the confidence intervals obtained applying the traditional LC independently for each population.

Table 2- Confidence Intervals for kt, $80 \%$ and $95 \%$, UK

Table 3- Confidence Intervals for kt, $80 \%$ and $95 \%$, Belgium

Table 4- Confidence Intervals for kt, $80 \%$ and $95 \%$, Spain

Table 5- Confidence Intervals for kt, $80 \%$ and $95 \%$, France

Table 6- Confidence Intervals for kt, $80 \%$ and $95 \%$, Italy

As is shown in Tables 2-6, the MLCPS produces confidence intervals which are wider than those obtained when applying separately the LC to each population. The result is due to the fact that, in the former case, more information is included in the model; in other words, the mortality is affected 
not only by national factors, but also by international factors. Of course, we refer to latent factors and not specific causes of death. However, adding information produces more effective results: if the mortality is influenced by national and international factors, the confidence intervals of the projections become wider.

\section{Concluding Remarks}

Several models have been developed for representing future mortality trends. In particular, the literature suggests that extrapolative methods lead to the most reliable approach in terms of forecast accuracy (Booth and Tickle, 2008). Among the class of models under consideration, we base our work on the LC model, because of its desirable features (Tuljapurkar et al., 2000). Therefore, in this paper we try to develop a more accurate algorithm in terms of prediction intervals. In order to do this, an improved predictor should take into account not only the dependence across age and time (D'Amato et al., 2012b), but also the dependence structure across different populations characterized by similar features, which are potentially affected by common factors.

We present an algorithm that preserves the parametric structure in a LC setting, as well as improves the goodness of fit of the predictor, because it has been constructed to take advantage of the appropriate tools for the bootstrap simulation of dependent data.

The analysis can be further extended to the study of possible convergence in some actuarial measures; for example, even though there is a convergence in the fitting of the mortality rates, life expectancy forecasts for population may diverge or not. Even if empirically the divergence may be small, from a theoretical point of view the forecasts of death rates and life expectancies may still diverge, because the beta parameters for different populations are different. One proposal to overcome this problem would be to consider that, for biological reasonableness, the ratio mxt,i / mxt, j should not diverge as $t$ tends to infinity as advocated by Cairns et al (2011). In this paper, we do not impose any restrictions to ensure that divergence is ruled out. Some restrictions could be imposed, the simplest one being that the betas are equal across populations. This would mean that the model becomes a nonstationary panel regression with one time effect; however, verifying the validity of such a restriction is in general not easy (see e.g. Castagnetti et al., 2013, for the case of stationary data). We, therefore, choose to leave the model unrestricted, pointing out that whilst long-term forecasts may have the implausible property that predictions for the same age $\mathrm{x}$ diverge across individual populations, this is not an issue for short term forecasting. Moreover, also in the long run, the empirical evidence suggested that that in a cluster of different population characterized by common geographical and socio-economic features mortality can be modelled and forecast coherently using a common structure (Hatzopoulos and Haberman (2013)).

Other interesting research questions are worth exploring. Whilst the presence of cross sectional dependence is considered in our framework, the standard LC model can be extended to accommodate for the presence of several common stochastic trends (as opposed to only one) and to include the presence of stationary common factors in the error term. The latter extension, in particular, could prove useful in order to take into account the presence of strong cross sectional dependence across units (i.e. across countries and age groups). This is a very important topic, since the bootstrap algorithm proposed here, per se, is a "one cross sectional unit at a time" algorithm, which ensures consistency only in the presence of weak cross dependence. Trapani (2013) discusses some extensions of the bootstrap theory to the case of multiple, stationary and non-stationary common trends. The application of such theory to the context of the LC model is currently under investigation by the authors. 


\section{References}

Anderson, T. W., 1984, An Introduction to Multivariate Statistical Analysis, 2nd Edition, New York: Wiley

Bai, J., 2004, Estimating cross-section common stochastic trends in nonstationary panel data, Journal of Econometrics, 122, 137-183

Booth, H., Maindonald J., Smith, L., 2002, Age-time interactions in mortality projections: applying Lee-Carter to Australia, Population Working Papers in Demography n. 85

Booth, H., Tickle, L., 2008, Mortality modelling and forecasting: a review of methods. Annals of Actuarial Science, 3, 3-43.

Bühlmann, P., 1997, Sieve bootstrap for time series, Bernoulli, 3, 123-148

Cairns et al 2011 is missing

Castagnetti, C., Rossi, E., Trapani, L., 2013. Inference on factor structures in heterogeneous panels. Mimeo.

D’Amato V., Di Lorenzo E., Haberman S., Russolillo M., Sibillo M., 2011, The Poisson logbilinear Lee Carter model: Applications of efficient bootstrap methods to annuity analyses. North American Actuarial Journal, 15(2), 315-333.

http://www.soa.org/library/journals/north-american-actuarial-journal/2011/no-2/naaj-2011-vol15no2.aspx

D’Amato V., Haberman S., Russolillo M., 2012a, The Stratified Sampling Bootstrap: an algorithm for measuring the uncertainty in forecast mortality rates in the Poisson Lee-Carter setting. Methodology and Computing in Applied Probability, 14(1), 135-148.

D’Amato V., Haberman S., Piscopo G., Russolillo M., 2012b, Modelling dependent data for longevity projections. Insurance Mathematics and Economics 51, 694-701, DOI information: 10.1016/j.insmatheco.2012.09.008

Gaille S., Sherris M., 2011, Modelling Mortality with Common Stochastic Long-Run Trends. The Geneva Papers on Risk and Insurance - Issues and Practice, 36(4), 595-621.

Hainut D., 2012, Multidimensional Lee-Carter model with switching mortality processes. Insurance Mathematics and Economics, 50(2), 236-246.

Hatzopoulos P, Haberman, S (2013), 'Common mortality modelling and coherent forecasts - an empirical analysis of worldwide mortality data', Insurance: Mathematics and Economics, 52(2), p.320-337

Lazar, D., Denuit, M., 2009, A multivariate time series approach to projected life tables. Applied Stochastic Models in Business and Industry, 25, 806-823. 
Lee, R.D., L. R. Carter, 1992, Modelling and Forecasting U.S. Mortality. Journal of the American Statistical Association, 87, 659-671.

Li, N., R. Lee, 2005, Coherent mortality forecasts for a group of populations: An extension of the Lee-Carter method. Demography, 42(3), 575-594.

Li, J. S. H., Hardy, M. R., 2011, Measuring Basis Risk Involved in Longevity Hedges. North American Actuarial Journal, 15(2), 177-200.

Njenga, C.N., Sherris, M., 2011, Longevity Risk and the Econometric Analysis of Mortality Trends and Volatility. Asia-Pacific Journal of Risk and Insurance, 5(2), Art.2

Russolillo, M., Giordano, G., Haberman, S., 2011, Extending the Lee-Carter model: a three-way decomposition. Scandinavian Actuarial Journal, 2, 96-117.

Stevens, R., 2011, A time-dependent age-factor extension to the Lee- Carter model, Longevity 7 , Frankfurt

Trapani, L., 2012, On bootstrapping panel factor series - Extended version. Available at SSRN: http://ssrn.com/abstract=2062183

Trapani, L., 2013, On bootstrapping panel factor series. Journal of Econometrics, 172, 127-141.

Tuljapurkar, S, Li, N and Boe, C, 2000, A universal pattern of mortality decline in the G7 countries. Nature, 405, 789-792.

United Nations, 1998, World Populations Prospects: The 1996 Revision. New York: Population Division, United Nations.

White, K. M., 2002, Longevity Advances in High-income Countries, 1955-96. Population and Development Review, 28, 59-76.

Wilson, C, 2001, On the Scale of Global Demographic Convergence 1950-2000. Population and Development Review, 24, 593-600. 